\section{CHEST EXAMINATION}

By Wing-Commander R. R. TRAIL. J. \& A. Churchill, Ltd., London, 1943. Price 10s. 6d.

Recent strides in radiography of the chest have created the need for a general overhaul of the whole question of physical signs of chest disease. Much simplification should be possible, as many of the time-honoured signs have been proved unreliable.

Trail sets out to correlate the underlying lung pathology with the clinical signs and X-ray appearances. He discusses the applied anatomy of the lungs and bronchi, and deals at some length with pathology as it occurs in the various morbid states. He sums up the clinical findings and the conclusions to be drawn in a section on physical signs, the final section being devoted to the appearance and technique in interpretation of the abnormal X-ray film.

Great emphasis is laid on a division of the inspiratory phase of the breath-sound into three parts. On auscultation an idea of the underlying pathology can be obtained from the intensity of the various adventitious sounds, and more especially the particular part of inspiration in which they occur. Thus there is said to be a stethoscopic difference between pneumonia and bronchopneumonia; between lung abscess and bronchiectasis with lung abscess; between generalised fibrosis and marked peribronchial fibrosis. In the very short section on physical examination much stress is placed on the importance of the sterno-mastoid sign. This is said to be the most constant sign of collapse in all its forms, and more reliable even than screening. Displacement of the apex-beat is mentioned only to be dismissed as "inconstant" or "even misleading."

Quite apart from the above there are many departures from the orthodox. For instance, it is stated that collapse of the right middle lobe produces displacement of the heart. Many will not agree with the assertion that the branching of the bronchial tree is dichotomous. Nor will it be generally accepted that fibrosis produces, widespread rales throughout inspiration remaining after cough. No references are given, even for such a statement as, in speaking of chronic bronchitis, "the vessels in the connective tissue, branches of the bronchial artery, suffer contraction up to endarteritis; this, together with increased resistance to the maintenance of oxygenation tends to produce hypertension."

The diagrams are clear, and the X-ray plates are good. The example of collapsed right middle is not a typical one, as in the lateral view it rather resembles interlobar flyid.

The book will be of considerable interest to those already experienced in chest diseases, representing as it does a new and original conception and interpretation of many of the everyday signs of chest illness. Although his methods are apparently of great assistance to the author, it is very likely that they wili prove confusing to the undergraduate student, and in some respects misleading.

\section{ALLERGY ANAPHYLAXIS AND IMMUNO- THERAPY}

By Bret Ratner, M.D., Baillière, Tindall \& Cox, London. 1943. Price $47 \mathrm{~s}$.

The author considers serum sickness the earliest and most easily identifiable manifestation of allergy, and that its complete study would give one the

\title{
Modern
} FOCAL THERAPY

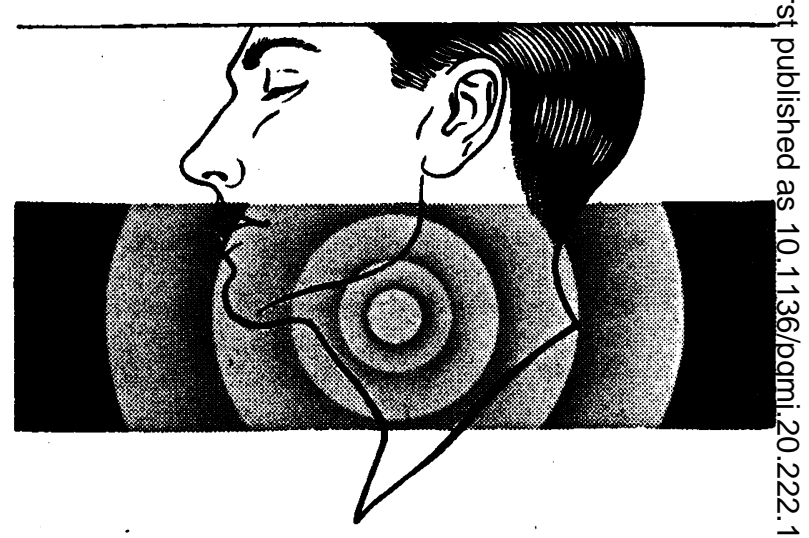

Fifty years ago Niels Finsen first tried to kills bacteria under the skin by shooting them with ultra-o violet rays. He achieved brilliant and epoch-makingresults although his hypothesis was wrong; for the ultra-violet rays when applied topically to the skin tissues, membranes, eliminate infections not throughe their bactericidal action so much as through the loesar immunizing, activating, and regenerative effegtso which they induce. In Finsen's time this therapy was a domain for specialists, exploring untrodden paths, using unwieldy equipment, devoting uncounted time. To-day focal ultra-violet irradiation with the Hanovia Kromayer Lamp is a well-defined therapy useful to every practitioner, applicable in any con sulting room, requiring little, time, and giving out standing results in nose, throat, skin, G.U., gynae cological and surgical conditions.

To investigate these results for yourself, ask uṣ for a copy of the Hanovia handbook "Modern FocaB Therapy" (48 pages, Io illustrations). It is free to professional enquirers.

This is one item in the all-round service (thera $\frac{D}{2}$ peutic information, supply, and maintenance of equipment, etc.) maintained by

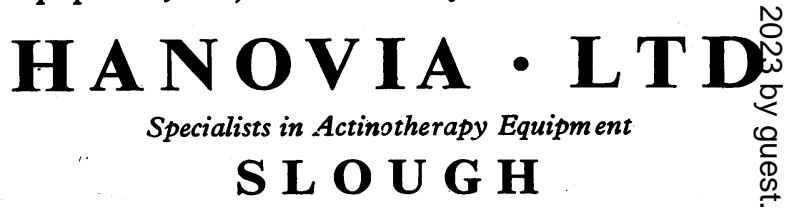

London Showrooms: 3, Victoria Street, S.W.I 